\title{
Procesos de enseñanza-aprendizaje en la universidad: perspectiva de los estudiantes
}

\author{
José Fernando Cardona Henao *
}

Magíster en Educación. Especialista en Pedagogía. Licenciado en Educación. Docente, Facultad de Ciencias Económicas y Contables. Universidad Cooperativa de Colombia, Pereira. Correo electrónico: jose.cardona@ campusucc.edu.co, jofeca6@yahoo.es

Recibido: 30 de julio del 2016

Aprobado: 22 de agosto del 2016

Cómo citar este artículo: Cardona, José Fernando. "Procesos de enseñanzaaprendizaje en la universidad: perspectiva de los estudiantes". Rastros Rostros 18.33 (2016): xx-xx. Impreso. doi: http://dx.doi. org/10.16925/ra.v18i33.1720

\section{Resumen}

Introducción: la necesidad de pasar de la enseñanza al aprendizaje, hace reflexionar el tema en aulas universitarias desde las exigencias de la educación para el siglo XXI, lo que reclama estrategias didácticas con procesos abiertos, flexibles e interdisciplinares. Desde una investigación descriptiva con unidad de trabajo de 1000 estudiantes de pregrado de tres universidades pereiranas, una muestra probabilística aleatoria simple de 100 estudiantes de ambos sexos, 30 de UniCatólica, 30 de UniAndina y 40 de UniLibre, a quienes se aplicó cuestionario Likert, para medir la enseñanza desde tres funciones específicas: a partir de la experimentación y el trabajo colaborativo; desde la correcta expresión y gesticulación del docente y de la motivación al estudiante, y a partir de buenas relaciones interpersonales y del control al aprendizaje del estudiante, cada función fue valorada desde diez indicadores. Resultados: se encontró que en la universidad privada pereirana, en programas de pregrado, la enseñanza-aprendizaje ofrecida es un proceso efectivo, dinámico y proactivo desde la perspectiva del estudiante y desde el análisis hecho a las metodologías y estrategias didácticas utilizadas por los docentes. Conclusiones: en la universidad pereirana, en programas de pregrado, se ofrece enseñanza-aprendizaje a partir de la experimentación y del trabajo colaborativo grupal; desde una correcta expresión y gesticulación del docente y de la motivación del estudiante, a partir de buenas relaciones interpersonales y control al aprendizaje del estudiante. De igual manera, se observa que en dichas instituciones la enseñanza-aprendizaje es un proceso efectivo, dinámico y proactivo desde la perspectiva del estudiante.

Palabras clave: aprendizaje, educación, enseñanza, estudiante, universidad. 


\title{
Teaching-learning processes in the university: students' perspective
}

\begin{abstract}
Introduction: the need to move from a teaching to a learning perspective makes the reflection appear in university classrooms from the education requirements for the twenty-first century, which calls for didactic strategies with open, flexible and interdisciplinary processes. From a descriptive research with a work unit of 1000 undergraduate students from three universities of Pereira, Colombia, a random probability probabilistic sample of 100 students of both sexes (30 from UniCatólica, 30 from UniAndina and 40 from UniLibre), to whom a Likert questionnaire was applied, was carried out to measure teaching from three specific functions: 1) experimentation and collaborative work; 2) the correct teacher's expression and gesture, and motivation to the student; 3) good interpersonal relationships and control of student learning. Each function was evaluated with ten indicators. Results: it was found that in the private universities of Pereira in undergraduate programs, the teaching-learning offered is an effective, dynamic and proactive process from the student's perspective and from the analysis done to the didactic methodologies and strategies applied by teachers. Conclusions: in the universities of Pereira, in undergraduate programs, teaching-learning is offered based on experimentation and group collaborative work; From correct teachers' expression and gesticulation as well as motivation of the student, from good interpersonal relations and control of the student's learning. In the same way, it became evident that in these institutions, teaching-learning is an effective, dynamic and proactive process from the student's perspective.
\end{abstract}

Keywords: learning, education, teaching, student, university.

\section{Processos de ensino-aprendizagem na universidade: perspectiva dos estudantes}

\section{Resumo}

Introdução: a necessidade de passar do ensino à aprendizagem, faz com que o tema reflexione em salas universitárias a partir das exigências da educação para o século XXI, o que pede estratégias didáticas com processos abertos, flexíveis e interdisciplinares. A partir de uma investigação descritiva com unidade de trabalho de 1000 estudantes de graduação de três universidades de Pereira, separou-se uma amostra probabilística aleatória simples de 100 estudantes de ambos os sexos (30 da UniCatólica, 30 da UniAndina e 40 da UniLibre), aos quais foi aplicado o questionário Likert para medir o ensino em três funções específicas: a experimentação e o trabalho colaborativo; a correta expressão e gesticulação do docente; e a motivação ao estudante e, baseado em boas relações interpessoais e no controle da aprendizagem do estudante, cada função foi valorizada partindo de dez indicadores. Resultados: descobriu-se que na universidade privada de Pereira, em programas de graduação, o ensino-aprendizagem oferecido é um processo efetivo, dinâmico e proativo segundo a perspectiva do estudante e a análise feita das metodologias e estratégias didáticas utilizadas pelos docentes. Conclusões: na universidade de Pereira, em programas de graduação, oferece-se ensino-aprendizagem a partir da experimentação e do trabalho colaborativo grupal; de uma correta expressão e gesticulação do docente; e da motivação do estudante, segundo boas relações interpessoais e controle da aprendizagem do estudante. Da mesma maneira, observa-se que em tais instituições o ensino-aprendizagem é um processo efetivo, dinâmico e proativo de acordo com a perspectiva do estudante.

Palavras-chave: aprendizagem, educação, ensino, estudante, universidade. 


\section{Introducción}

El proyecto nace de la preocupación de docentes de la Especialización en Docencia Universitaria, en la línea de investigación en Pedagogía y Currículo, allí surge el interrogante sobre la eficiencia y eficacia de los procesos enseñanza-aprendizaje brindados en aulas universitarias privadas, inquietud que se ha convertido en el horizonte de dicha especialización. Desde allí se vio la necesidad de analizar la efectividad del proceso en la ciudad de Pereira, un reto desde cualquier perspectiva, y en especial, si se hace a la luz de las nuevas exigencias de la educación en el siglo XxI, que reclama el paso de la enseñanza al aprendizaje, para lo cual se requieren nuevas estrategias didácticas. El trabajo encuentra justificación no solo en lo planteado sino también en el hecho que a nivel regional el tema ha sido poco estudiado, y que son los estudiantes quienes tienen la última palabra, lo que lleva a que las implicaciones prácticas del proceso investigativo sean de alto beneficio no solo para docentes y directivos, quienes tienen en sus manos la planeación de la enseñanza-aprendizaje sino para la comunidad académica pereirana, la cual desde los resultados podría asumir acciones concretas para el mejoramiento de la educación superior.

Teniendo presente el razonamiento anterior y el hecho de que la universidad tiene como función formar la masa crítica del país, objetivo que solo se logra con procesos abiertos, flexibles e interdisciplinares, que en la academia de hoy, difícilmente se dan, pues aún prevalecen metodologías de enseñanza y no de aprendizaje, centradas en el docente y no en el estudiante, se quiso conocer la opinión de estudiantes de pregrado de las universidades Católica, Libre, y Andina, sobre la efectividad y calidad de la enseñanza recibida. Para ello se aplicó el cuestionario Likert que mide la enseñanza desde tres funciones específicas: enseñanza a partir de la experimentación y el trabajo colaborativo; enseñanza a partir de una correcta expresión y gesticulación del docente y de la motivación al estudiante; y enseñanza a partir de las buenas relaciones interpersonales y del control al aprendizaje del estudiante. Cada función fue valorada desde diez indicadores.

Dicha investigación descriptiva llevó a concluir que en la universidad privada pereirana, en los pregrados, la enseñanza-aprendizaje que se ofrece es un proceso efectivo, dinámico y proactivo desde la perspectiva del estudiante y desde el análisis hecho a las metodologías y estrategias didácticas utilizadas por los docentes que orientan cursos en dichas universidades objeto de estudio. De igual manera, se llegó a entender que la universidad juega papel preponderante en la educación y formación de futuros profesionales y dirigentes sociales, lo que le reclama la búsqueda de estrategias de mejoramiento de procesos pedagógicos, didácticos y evaluativos, centrados en el estudiante.

\section{Metodología}

Hoy, enseñar se hace cada vez más complejo, aprender se convierte en experiencia mucho más desafiante para el aprendiz, no obstante, cada día hay más y mayor convencimiento que la educación es el camino más viable para hacer posible la paz y la convivencia ciudadana, por ello, trabajar en el desarrollo de competencias ciudadanas es tomar la decisión de "hacer la democracia en el país" (Chaux 17), de formar ciudadanos comprometidos, respetuosos de la diferencia y defensores del bien común. De ahí, que la función de la educación sea formar personas, acrecentar lo humano, lo espiritual y lo social, esta ha de ser la misión de todo educador y a esto debe apostarle una sociedad amenazada desde la economía, la política, la tecnología, la globalización y también por la misma educación, ya que está determinada por currículos agregados que desconocen el tiempo, el espacio, las circunstancias y la historia especifica de la comunidad a la que van dirigidos, quedando sólo en un agregado de áreas y asignaturas, sin coherencia ni interdisciplinariedad, pero para lograr hacer bien la tarea, la educación requiere según Giroux: "Docentes transformadores, que vivan los aprendizajes como prácticas liberadoras, lúdicas y creativas, al igual que de instituciones que reflejen la cultura desde la sensibilidad hasta la autoformación y autovaloración, con un discurso para la equidad y el mutuo reconocimiento" (112).

La universidad juega un papel preponderante en la educación y formación de futuros profesionales y dirigentes sociales, lo que le reclama la búsqueda de estrategias de mejoramiento de procesos pedagógicos, didácticos y evaluativos, centrados en el estudiante, de ahí, que:

Urge por tanto facilitar habilidades cognitivas y culturales que ayuden a descodificar (leer) y codificar (expresar) con registros alternativos que rompan con la pasividad del consumidor, incapaz de controlar el proceso de tecnologización de la sociedad de la información y de las claves culturales con las que viene expresada la cultura escolar. (Antón 60) 
¿La universidad colombiana tiene como prioritaria esta tarea?, la cual si es bien hecha, lleva a formar ciudadanos capaces de crear lazos de solidaridad, espacios de participación y generar normas de sana convivencia. Ciudadanos seguros de sí mismos y confiados en los otros; capaces de analizar y aportar en procesos colectivos; que prefieran el acuerdo y el pacto antes que las armas para resolver conflictos.

Este razonamiento llevó a realizar una investigación descriptiva, con una unidad de trabajo de 1000 estudiantes aproximadamente, de programas de pregrado de universidades privadas pereiranas. Se extrae una muestra probabilística aleatoria simple de 100 estudiantes: 30 de la Universidad Católica; 30 de la Universidad Andina y 40 de la Universidad Libre, a quienes se aplica un cuestionario tipo Likert para medir la enseñanza recibida desde tres funciones básicas, de acuerdo con Martínez: "Enseñanza a partir de la experimentación y el trabajo colaborativo; enseñanza a partir de una correcta expresión y gesticulación del docente y de la motivación al estudiante; y una enseñanza a partir de las buenas relaciones interpersonales y del control al aprendizaje del estudiante" (48), cada función fue valorada desde diez indicadores. Las tablas 1,2 y 3 muestran los resultados.

Tabla 1. Generalidades población objeto de estudio. Unidad de análisis

\begin{tabular}{|c|c|c|c|c|c|c|c|}
\hline \multirow[t]{2}{*}{ N. ${ }^{\circ}$} & \multicolumn{4}{|c|}{ SEXO } & \multirow[t]{2}{*}{ UNIVERSIDAD } & \multirow[t]{2}{*}{ TOTALES } & \multirow[t]{2}{*}{$\%$} \\
\hline & $\mathrm{M}$ & $\%$ & $\mathrm{~F}$ & $\%$ & & & \\
\hline 1 & 14 & 46,6 & 16 & 53,3 & Católica de Pereira & 30 & $30 \%$ \\
\hline 2 & 13 & 43,3 & 17 & 56,6 & Universidad Andina & 30 & $30 \%$ \\
\hline 3 & 21 & 52,5 & 19 & 47,5 & Universidad Libre & 40 & $40 \%$ \\
\hline Total & 48 & $48 \%$ & 52 & $52 \%$ & & 100 & $100 \%$ \\
\hline
\end{tabular}

Fuente: elaboración propia

Tabla 2. Fuentes de información

\begin{tabular}{|c|c|c|c|}
\hline FUENTE & TIPO & QUIENES & OBJETIVO \\
\hline Primaria & $\begin{array}{l}\text { Cuestionario } \\
\text { Likert }\end{array}$ & $\begin{array}{l}\text { Estudiantes de pregrado } \\
\text { universidades privadas de } \\
\text { la ciudad de Pereira }\end{array}$ & $\begin{array}{l}\text { Describir la efectividad del proceso enseñanza - aprendi- } \\
\text { zaje llevado a cabo en instituciones de educación superior } \\
\text { privadas de la ciudad de Pereira. }\end{array}$ \\
\hline \begin{tabular}{|l|} 
Secundarias \\
\end{tabular} & $\begin{array}{l}\text { Indicadores de la fun- } \\
\text { ción didáctica } \\
\text { Enseñanza aprendizaje } \\
\text { en la educación superior }\end{array}$ & $\begin{array}{l}\text { Enrique Martínez-Sala- } \\
\text { nova Sánchez. } \\
\text { Silvia Arce, Coordinadora } \\
\text { Área Psicopedagógica } \\
\text {-FACED }\end{array}$ & $\begin{array}{l}\text { Describir la efectividad del proceso enseñanza-aprendi- } \\
\text { zaje llevado a cabo en instituciones de educación superior } \\
\text { privadas de la ciudad de Pereira. }\end{array}$ \\
\hline
\end{tabular}

Fuente: elaboración propia.

En el mundo globalizado, en esta "aldea planetaria” (Morín 45), es necesario que la educación propenda por formar hombres y mujeres, capaces de enfrentar el desafío de una nación que hay que rehacer, estudiantes universitarios que vean con lucidez qué es lo que deben hacer y no duden en obrar con responsabilidad social y autenticidad.

A la universidad corresponde la responsabilidad de formar ciudadanos útiles, pero esta posibilidad incluye trabajar el concepto de formación, que desde Gadamer es concebida como: "Construcción comprensiva, multicultural, democrática, de búsqueda, de negociación, de crecimiento, de confrontación entre la cultura universal y la cultura de lo cotidiano" (40).
Para lo cual, se ha de asumir la docencia como una relación social pedagógica con intencionalidad especifica de formación integral, en la que cada maestro contribuya a que el estudiante cultive sus potencialidades y competencias para el reconocimiento de los problemas, la búsqueda de soluciones alternativas y su aplicación en la transformación social. Corresponde a la comunidad universitaria, con sus docentes a la cabeza, un reflexionar epistémico y sistémico sobre el quehacer universitario bajo el imperativo de quienes somos en la sociedad colombiana, según Tobón: "Los escenarios de vida son inciertos gracias a la miseria, a la pobreza, a la muerte, a la violencia y a la destrucción de los recursos naturales" (112), y es desde aquí 
que ha de hablarse de la efectividad del proceso enseñanza-aprendizaje en la educación superior, lo que supone un caminar por investigaciones que puedan llevar a lograr un acercamiento a la temática.

Tabla 3. Variables e indicadores

\begin{tabular}{|l|l|l|l|}
\hline \multicolumn{1}{|c|}{ VARIABLE } & \multicolumn{1}{|c|}{ DEFINICIÓN } & \multicolumn{1}{c|}{ INDICADORES } & \multicolumn{1}{c|}{ OBJETIVO } \\
\hline $\begin{array}{l}\text { Experimentación y } \\
\text { trabajo colaborativo }\end{array}$ & $\begin{array}{l}\text { Proceso de enseñanza basado en } \\
\text { el uso de la experiencia, el expe- } \\
\text { rimento, la reflexión y el trabajo } \\
\text { en equipo. }\end{array}$ & $\begin{array}{l}\text { Contenidos } \\
\text { Actividades } \\
\text { Técnicas de trabajo } \\
\text { Investigación } \\
\text { Asesoría } \\
\text { Trabajo en equipo } \\
\text { Resultados }\end{array}$ & $\begin{array}{l}\text { Analizar enseñanza a partir de la } \\
\text { experimentación y el trabajo colabo- } \\
\text { rativo en los programas de pregrado } \\
\text { en las universidades privadas de la } \\
\text { ciudad de Pereira. }\end{array}$ \\
\hline $\begin{array}{l}\text { Expresión docente y } \\
\text { motivación al estu- } \\
\text { diante }\end{array}$ & $\begin{array}{l}\text { Proceso de enseñanza basado } \\
\text { en la correcta y justo trato del } \\
\text { docente, así como en la incenti- } \\
\text { vación permanente al estudiante. }\end{array}$ & $\begin{array}{l}\text { Recursos didácticos } \\
\text { Ambiente de clase } \\
\text { Recapitulaciones } \\
\text { Interés } \\
\text { Temas } \\
\text { Atención } \\
\text { Actitud docente }\end{array}$ & $\begin{array}{l}\text { Analizar enseñanza a partir de la } \\
\text { correcta expresión docente y de la } \\
\text { motivación al estudiante en los pro- } \\
\text { gramas de pregrado en las universida- } \\
\text { des privadas de la ciudad de Pereira. }\end{array}$ \\
\hline $\begin{array}{l}\text { Control del aprendizaje } \\
\text { y relaciones interper- } \\
\text { sonales }\end{array}$ & $\begin{array}{l}\text { Proceso de enseñanza basado } \\
\text { en el seguimiento continuo a } \\
\text { los progresos o avances de los } \\
\text { estudiantes y en un clima de clase } \\
\text { ameno, agradable y cordial. }\end{array}$ & $\begin{array}{l}\text { Orientaciones } \\
\text { Confianza } \\
\text { Retroalimentación } \\
\text { Comprensión } \\
\text { Aprendizaje } \\
\text { Estrategias }\end{array}$ & $\begin{array}{l}\text { Analizar enseñanza a partir del con- } \\
\text { trol del aprendizaje del estudiante y de } \\
\text { las buenas relaciones interpersonales } \\
\text { en los programas de pregrado en las } \\
\text { universidades privadas de la ciudad } \\
\text { de Pereira. }\end{array}$ \\
\end{tabular}

Fuente: elaboración propia.

En su investigación Los nuevos desafíos de la didáctica de la educación superior en el siglo XXI (50), Moreno plantea problemas teóricos que caracterizan la situación actual de la didáctica general y las didácticas especiales, como la influencia que ha tenido el concepto de currículum que en ocasiones ha absorbido o subsumido a la didáctica, así como las luchas de los profesionales por el control de la enseñanza y el debilitamiento del discurso de la pedagogía. En la mencionada investigación se arriba a la necesidad de reconstruir el discurso de la didáctica y de la pedagogía, visualizando la necesidad de adaptar el discurso y la acción didáctica a las nuevas demandas que se plantean las instituciones de educación superior en el siglo xxI, especifica que dado que la didáctica tiene como foco de interés el proceso de enseñanza-aprendizaje, sólo se le podrá entender en relación con dicho proceso.

Guzmán, en su investigación Calidad de la enseñanza en educación superior (43), aclara que el concepto de calidad docente despierta debates, ya que para caracterizar a un buen profesor es necesario ir más allá de un listado de habilidades de enseñanza, pues la calidad es más que una descripción de destrezas independientes, y también señala que hay que tomar en cuenta algunos aspectos como: identidad profesional, creencias pedagógicas y compromiso con la profesión.

Respecto a la evaluación docente, CochranSmith afirma que "aunque las evaluaciones realizadas por los alumnos son parte importante para juzgar el desempeño docente, no debe ser el único criterio, y sugiere incluir otros elementos como la búsqueda, por parte del docente, de la equidad y la justicia social" (14). Para el autor, los buenos maestros y las buenas escuelas no pueden por sí solos arreglar las desigualdades e inequidades sociales. Precisa que lo anterior no puede usarse como excusa para que los educadores se desliguen de su responsabilidad en el aprendizaje de los estudiantes. De ahí que el reto, de acuerdo con Cochran-Smith, es "crear ambientes que ofrezcan variadas oportunidades de aprendizaje para los alumnos, y para eso se necesitan profesores que sepan proveer las oportunidades para que todos lo logren" (16).

\section{Resultados}

Los procesos de enseñanza y aprendizaje han estado y estarán en tela de juicio, en especial cuando los 
estudiantes no logran buenos aprendizajes o lo que hoy se denomina aprendizajes significativos. Para iniciar esta discusión es conveniente hacerlo desde lo que plantea Arce, quien dice que: "A través de la historia, la educación se ha centrado en transmitir el conocimiento; viendo a los educandos como un recipiente que el docente llene de información. En dicho enfoque, el estudiante es un simple receptor y el docente un constatador de cuánto se le dio al estudiante y de cuanto es capaz de repetir de memoria; es decir, que los docentes se han dedicado a explorar la capacidad memorística del estudiante, además de formarlos para que compitan entre sí para alcanzar las mejores calificaciones, en lugar de formar personas capaces de cooperar para lograr un fin común" (56). Es la comúnmente llamada educación bancaria. Desde esta premisa se analizarán los siguientes resultados de investigación, teniendo presente que es el profesor, "quien requerirá aprender, desaprender y reaprender nuevas competencias" (Stoll, Fink y Earl 43).

Respecto a la función 1 (tabla 4), relacionada con el análisis de la enseñanza-aprendizaje de procesos de experimentación, simulación, contextualización, trabajo colaborativo y cooperativo, se aprecia que los indicadores utilizados para medir dicha función favorecen la labor docente en el aula, y el proceso educativo llevado a acabo. Los estudiantes están de acuerdo en afirmar que se les brinda enseñanza a partir de la experimentación y el trabajo en equipo. Indicadores como la preparación de contenidos y materiales para la clase, el seguimiento individual a las actividades de los estudiantes y el brindar instrucciones claras y concretas para el desarrollo del trabajo en clase, obtienen altas valoraciones, sobrepasando el 95\% del total, sumando las alternativas siempre y casi siempre. De igual manera, y en su orden, los indicadores énfasis en resultados y aclaraciones una vez terminado el trabajo en clase, la estimulación para la producción de ideas por parte del estudiante y el brindar información suficiente sobre técnicas de trabajo y para búsqueda de información, alcanzan bajas valoraciones, y aunque se han mantenido por encima del $90 \%$, merecen atención y mejoramiento.

Tabla 4. Función 1, enseñanza-aprendizaje a partir de la experimentación y el trabajo colaborativo

\begin{tabular}{|l|l|c|c|c|c|c|c|c|c|c|}
\hline N. $^{\circ}$ & \multicolumn{1}{|c|}{ Afirmación } & S & $\%$ & CS & $\%$ & PV & $\%$ & N & $\%$ & Totales \\
\hline 1 & Preparación contenidos y materiales & 38 & 38 & 60 & 60 & 1 & 1 & 1 & 1 & 100 \\
\hline 2 & Seguimiento actividades estudiante & 32 & 32 & 62 & 62 & 6 & 6 & 0 & 0 & 100 \\
\hline 3 & Utilización experiencias estudiantes & 23 & 23 & 75 & 75 & 2 & 2 & 0 & 0 & 100 \\
\hline 4 & Información de técnicas de trabajo & 22 & 22 & 72 & 72 & 6 & 6 & 0 & 0 & 100 \\
\hline 5 & Promoción de la investigación & 24 & 24 & 70 & 70 & 5 & 5 & 1 & 1 & 100 \\
\hline 6 & Instrucciones claras y concretas & 32 & 312 & 65 & 65 & 3 & 3 & 0 & 0 & 100 \\
\hline 7 & Tiempo para trabajo en clase & 26 & 26 & 69 & 69 & 5 & 5 & 0 & 0 & 100 \\
\hline 8 & Asesorías constantes al estudiante & 25 & 25 & 69 & 69 & 6 & 6 & 0 & 0 & 100 \\
\hline 9 & Enfatiza resultados y aclaraciones & 15 & 15 & 77 & 77 & 8 & 8 & 0 & 0 & $1 \mid 00$ \\
\hline 10 & Estimula producción de ideas & 20 & 20 & 72 & 72 & 8 & 8 & 0 & 0 & 100 \\
\hline Totales & & 257 & 25,7 & 691 & 69,1 & 50 & 5 & 2 & 0,2 & 1000 \\
\hline
\end{tabular}

Siempre (S); Casi siempre (CS); Pocas veces (PV); Nunca (N).

Fuente: elaboración propia

En términos generales, los estudiantes están de acuerdo en un $94 \%$ en afirmar que sus docentes brindan enseñanza fundamentada en procesos de experimentación y trabajo en equipo, lo cual es una necesidad apremiante hoy para los futuros profesionales, y de paso facilita y motiva los aprendizajes. Un porcentaje muy pequeño representado en el 5\% de los encuestados afirman que esos procesos de enseñanza-aprendizaje se dan pocas veces y para el 0,2\% nunca. Teniendo en cuenta el análisis hecho, puede decirse que los docentes de las tres universidades tomadas como unidad de análisis, en los programas de pregrado, utilizan metodologías y estrategias didácticas basadas en la experimentación y fortalecen el trabajo en equipo, con lo que el aprendizaje que obtiene el estudiante es significativo y aplicable a contextos reales de la vida cotidiana. De igual manera, en este proceso de enseñanza, el docente utiliza la experiencia y conocimiento de los estudiantes en el desarrollo de las clases, hace seguimiento a las actividades del estudiante y brinda según encuestadas asesorías permanentes. 
Dos indicadores merecen especial atención, y requieren planes de mejoramiento, ya que si se logra elevar su efectividad el aprendizaje será aún más significativo y redundará en el mejoramiento de la calidad de formación recibida por el educando. Se hace referencia aquí a la necesidad de fortalecer durante la clase el énfasis en los resultados del trabajo realizado y brindar las aclaraciones suficientes y oportunas según sea el caso. De igual manera, el estimular la producción de ideas propias de los estudiantes durante las clases, es apremiante.

La tabla 5 muestra indicadores de medición para la función 2, enseñanza a partir de una correcta expresión y gesticulación docente y a partir de la motivación constante al estudiante. Se observa como la labor docente es efectiva. Los resultados muestran como indicadores relacionados con estrategias para despertar el interés del educando, el tono y volumen de voz y la creación de ambientes dinámicos de clase, obtienen valoraciones muy altas que sobrepasan el 95\% del total de las alternativas siempre y casi siempre. De esta manera se facilita la comprensión y el aprendizaje del estudiante. Tres indicadores obtienen valoraciones bajas, pero se ubican por encima del $90 \%$. En su orden, estos indicadores se relacionan con la necesidad que el docente realice síntesis y recapitulaciones al finalizar clase, con la urgencia que logre captar y mantener el interés y la motivación del estudiante durante el desarrollo de la clase y con la necesidad que se utilicen más ejemplos que permitan relacionar teorías estudiadas y su aplicación en contextos reales.

Tabla 5. Función 2, enseñanza-aprendizaje a partir de la correcta expresión y la motivación del estudiante

\begin{tabular}{|c|c|c|c|c|c|c|c|c|c|c|}
\hline N. ${ }^{\circ}$ & Afirmación & $S$ & $\%$ & $\mathrm{CS}$ & $\%$ & PV & $\%$ & $\mathrm{~N}$ & $\%$ & Totales \\
\hline 1 & Tono y volumen de voz = comprensión & 33 & 33 & 63 & 63 & 4 & 4 & 0 & 0 & 100 \\
\hline 2 & Utilización de recursos adecuados & 26 & 26 & 69 & 69 & 5 & 5 & 0 & 0 & 100 \\
\hline 3 & Creación de ambiente dinámico & 28 & 28 & 66 & 66 & 6 & 6 & 0 & 0 & 100 \\
\hline 4 & Destacan conceptos importantes & 21 & 21 & 68 & 68 & 11 & 11 & 0 & 0 & 100 \\
\hline 5 & Realiza síntesis y recapitulaciones & 17 & 17 & 71 & 71 & 12 & 12 & 0 & 0 & 100 \\
\hline 6 & Despierta el interés del estudiante & 35 & 35 & 59 & 59 & 6 & 6 & 0 & 0 & 100 \\
\hline 7 & Estimula participación del estudiante & 27 & 27 & 69 & 69 & 4 & 4 & 0 & 0 & 100 \\
\hline 8 & Relaciona teorías con ejemplos claros & 23 & 23 & 65 & 65 & 12 & 12 & 0 & 0 & 100 \\
\hline 9 & Sostiene atención del estudiante & 14 & 14 & 80 & 80 & 6 & 6 & 0 & 0 & 100 \\
\hline 10 & Actitud amistosa y acogedora en clase & 15 & 15 & 80 & 80 & 5 & 5 & 0 & 0 & 100 \\
\hline \multicolumn{2}{|c|}{ Totales } & 239 & 23,9 & 690 & 69,0 & 71 & 7,1 & 0 & 0 & 1000 \\
\hline
\end{tabular}

Siempre (S); Casi siempre (CS); Pocas veces (PV); Nunca (N).

Fuente: elaboración propia.

Como sucedió con la medición y análisis de la función 1, los resultados dejan ver que los estudiantes afirman, en un 92\%, que los docentes, en las tres universidades, ofrecen enseñanza fundamentada en correcta expresión y gesticulación, logrando con ello la motivación del estudiante para su aprendizaje. El $7 \%$ afirma que el tipo de enseñanza en mención se da pocas veces. No hay valoraciones para la alternativa nunca. El análisis lleva a concluir que la enseñanza ofrecida por los docentes, en programas de pregrado, es un proceso dinámico, asertivo y efectivo que gira en torno a la buena expresión y motivación estudiantil, lo que redunda en la efectividad del aprendizaje alcanzado por el educando. Dicha enseñanza está permeada por la utilización de recursos didácticos, adecuados a cada tema, de ambientes dinámicos de clase, de ejemplos que llevan a simular la aplicación de teorías en contextos reales y ante todo, es una enseñanza agradable, gracias a la actitud amistosa y acogedora que se vive en cada clase.

Como siempre, hay aspectos a mejorar, se requiere cuidado al hecho que el docente destaque conceptos importantes a través de resultados y aclaraciones al finalizar cada clase, pues con esta estrategia se mejoraría aún más el aprendizaje, y de paso, se aclaran dudas. De igual manera, se ha de fortalecer los ejemplos que faciliten la relación de teorías con contextos reales.

El análisis de la función 3, enseñanza-aprendizaje a partir de las buenas relaciones interpersonales y del control al aprendizaje del estudiante, muestra en la tabla 6 que, en su mayoría, los indicadores de medición obtienen valoraciones altas, sobrepasando el 95\% del total unificando alternativas siempre y casi 
siempre. De manera especial, indicadores relacionados con el hecho que el docente tiene en cuenta necesidades individuales del estudiante y las orienta; que el docente tiene organizado un sistema de retroalimentación para cada clase y que el docente hace preguntas y propone ejercicios para verificar la comprensión del estudiante; y que el docente brinda confianza al estudiante durante el desarrollo de la clase, muestran como los encuestados están de acuerdo en afirmar que sus maestros ofrecen una enseñanza, en la cual priman las buenas relaciones interpersonales y el seguimiento al control del aprendizaje, así como la confianza, elementos que favorecen cualquier aprendizaje.

Tabla 6. Función 3, enseñanza-aprendizaje a partir de buenas relaciones interpersonales y control al aprendizaje del estudiante

\begin{tabular}{|c|c|c|c|c|c|c|c|c|c|c|}
\hline N. ${ }^{\circ}$ & Afirmación & $S$ & $\%$ & $\mathrm{CS}$ & $\%$ & PV & $\%$ & $\mathbf{N}$ & $\%$ & Totales \\
\hline 1 & Orienta necesidades individuales & 44 & 44 & 50 & 50 & 6 & 6 & 0 & 0 & 100 \\
\hline 2 & Respeta la opinión de los demás & 29 & 29 & 67 & 67 & 4 & 4 & 0 & 0 & 100 \\
\hline 3 & Brinda confianza al estudiante en clase & 29 & 29 & 67 & 67 & 4 & 4 & 0 & 0 & 100 \\
\hline 4 & Valora conocimientos de estudiantes & 21 & 21 & 74 & 74 & 4 & 4 & 1 & 1 & 100 \\
\hline 5 & Evita diferencias al dirigirse a estudiante & 22 & 22 & 69 & 69 & 9 & 9 & 0 & 0 & 100 \\
\hline 6 & Existe retroalimentación para cada clase & 45 & 45 & 54 & 54 & 1 & 1 & 0 & 0 & 100 \\
\hline 7 & Verifica comprensión del estudiante & 35 & 35 & 63 & 63 & 2 & 2 & 0 & 0 & 100 \\
\hline 8 & Propone ejercicios complementarios & 29 & 29 & 65 & 65 & 6 & 6 & 0 & 0 & 100 \\
\hline 9 & Verifica recepción de estudiantes & 19 & 19 & 76 & 76 & 5 & 5 & 0 & 0 & 100 \\
\hline 10 & Reorienta estrategias para aprendizaje & 15 & 15 & 76 & 76 & 8 & 8 & 1 & 1 & 100 \\
\hline \multicolumn{2}{|c|}{ Totales } & 288 & 28.8 & 661 & 66.1 & 49 & 4.9 & 2 & 0.2 & 1000 \\
\hline
\end{tabular}

Siempre (S); Casi siempre (CS); Pocas veces (PV); Nunca (N).

Fuente: elaboración propia

Los demás indicadores de medición se mantienen por encima del $90 \%$, lo que de alguna manera fortalece la labor docente en el aula universitaria. Aquí, indicadores que señalan que existe retroalimentación para cada clase; el que hace mención sobre la verificación del grado de recepción del mensaje por parte del estudiante; y el que aduce que el docente valora los conocimientos y experiencias de los estudiantes, reflejan la buena aceptación de la labor docente por parte del estudiante, cliente a satisfacer.

Dos indicadores que, aunque aparecen por encima del $90 \%$ de valoración, reclaman atención especial ya que en ellos se observan porcentajes negativos visualizados en la alternativa, pocas veces, necesitan una revisión y fortalecimiento. Caso concreto del indicador que señala, que el docente evita dirigirse a unos estudiantes más que a otros evitando diferencias; y del indicador que menciona la necesidad que el docente modifique su ritmo de trabajo y reoriente su estrategia en función del aprendizaje del educando. En general, y de acuerdo con lo planteado en la función 3, los encuestados afirman que la enseñanza que se les brinda en la universidad, en programas de pregrado, es un proceso en el cual se tiene en cuenta como mecanismos para lograr mejores aprendizajes; las buenas relaciones interpersonales; y el control al seguimiento del aprendizaje. Así, y teniendo presente el análisis de las funciones que debe cumplir la enseñanza y desde la perspectiva de los estudiantes universitarios de pregrado, los docentes utilizan metodologías y estrategias didácticas que favorecen y facilitan el aprendizaje significativo de sus estudiantes.

\section{Conclusiones}

Todo proceso de enseñanza debe conducir al aprendizaje significativo y contextualizado de todos y cada uno de los aprendices participantes; por tal motivo, este proceso no puede darse el lujo de favorecer el aprendizaje en unos más que en otros. Corresponde al docente, desde sus metodologías y estrategias didácticas, llegar al estudiante y desarrollar en él competencias necesarias y suficientes para el saber conocer, el saber hacer y el saber ser y convivir, logrando de esta manera ser apoyo en la formación de personas de bien y profesionalmente capaces.

Desde esta perspectiva cabe anotar, que el proceso de enseñanza, para que sea efectivo, proactivo y eficaz, ha de cumplir tres funciones específicas: ser 
una enseñanza a partir de la experimentación y del trabajo colaborativo; una enseñanza a partir de una correcta expresión y gesticulación docente y a partir de la motivación constante del estudiante; y una enseñanza a partir de las buenas relaciones interpersonales y a partir del control al aprendizaje del estudiante.

En la universidad privada pereirana en los programas de pregrado, según ofrece enseñanza-aprendizaje a partir de la experimentación y del trabajo colaborativo (grupal); enseñanza a partir de una correcta expresión y gesticulación del docente y de la motivación del estudiante; enseñanza a partir de las buenas relaciones interpersonales y del control al aprendizaje del estudiante.

Respecto a la función 1, enseñanza a partir de la experimentación y del trabajo colaborativo, dos indicadores merecen especial atención, si de mejorar los aprendizajes se trata. Se hace referencia a dos hechos: el primero en relación con que el docente debe enfatizar más en resultados y aclaraciones una vez terminado el trabajo colaborativo y la sesión de clase; el segundo en función a que el docente ha de fortalecer estrategias y mecanismos para llevar al estudiante a producir sus propias ideas en cada sesión de clase.

La función 2, enseñanza a partir de una correcta expresión y gesticulación y de la motivación al estudiante, alcanza en su conjunto valoración por encima del 95\%, pero como siempre, en todo proceso hay aspectos dignos de mejorar. Para esta función, dos indicadores revisten atención y mejora, con lo cual de paso se mejora el aprendizaje: el primero relacionado con el hecho que el docente ha de destacar conceptos importantes a través de repeticiones adecuadas, favoreciendo la comprensión; y el segundo relacionado con que el docente emplee o utilice más y mejores ejemplos que permitan relacionar teorías y su aplicación en contextos reales.

En los indicadores de medición de la función 3, enseñanza a partir de las buenas relaciones interpersonales y del control al aprendizaje del estudiante, se observa que es la función con más alta valoración, llegando a sobrepasar el 96\% del total, pero de igual manera, en dicha función dos indicadores con la más baja valoración son dignos de atención y mejoramiento. Aquí, se hace un llamado al docente para que evite diferencias, dirigiéndose por igual a todos los participantes. El otro indicador que merece atención está relacionado con el hecho que el educador debe modificar su ritmo y reorientar sus estrategias cuando sea necesario para de esta manera facilitar el aprendizaje.

Como conclusión general puede afirmarse que en la universidad privada pereirana, en los programas de pregrado, la enseñanza-aprendizaje que se ofrece es un proceso efectivo, dinámico y proactivo desde la perspectiva del estudiante y desde el análisis hecho a las metodologías y estrategias didácticas utilizadas por los docentes que orientan cursos en dichas universidades objeto de estudio.

Es urgente que las instituciones de educación superior busquen estrategias de mejoramiento de procesos pedagógicos, didácticos y evaluativos, centrados en el estudiante y que redunden siempre en el mejoramiento de los aprendizajes del estudiante. Para ello, puede establecer planes y programas encaminados a:

Actualizary profesionalizar a los docentes no solo en sus áreas de formación, sino en estrategias pedagógicas y didácticas contextualizadas y de manera especial, formación en procesos de evaluación.

Institucionalizar estrategias como la escuela para la excelencia, en las que el docente permanentemente, se capacite y actualice en enseñanza- aprendizaje y evaluación por competencias.

Establecer programas como colectivos docentes o reflexiones pedagógicas por áreas de conocimiento, en los que se reflexione la praxis docente, se cuenten y socialicen experiencias de enseñanza aprendizaje entre docentes y se enriquezca en común dicha labor

Como el proceso enseñanza-aprendizaje nos es un proceso unilateral sino que requiere la actuación proactiva de docentes y discentes, al estudiante universitario le corresponde, de igual manera, aportar su grano de arena y desarrollar sus aspectos metacognitivos, si desea lograr aprendizajes significativos, aplicables y contextualizados. Esto lo puede hacer a través de estrategias que logren mejorar su responsabilidad, asistencia y puntualidad, dedicar al estudio el tiempo suficiente para responder a cabalidad con sus tareas y obligaciones, entender que su formación depende en un 50\% de su interés, de su motivación y de su responsabilidad, desarrollar competencias lecto-escriturales que le permitan mejorar su capacidad de comprensión, y análisis, pues muchos estudiantes universitarios tiene falencias en estos aspectos lo que dificulta su aprendizaje y proponer nuevas estrategias y formas de enseñanza que favorezcan su aprendizaje, más que la enseñanza 


\section{Referencias}

Antón, José Antonio. "La pedagogía crítica desde la perspectiva de los movimientos sociales". Tabanque. Revista Pedagógica 17 (2011): 51-70. Impreso.

Arce, Silvia. El proceso de enseñanza-aprendizaje en la educación superior. Galileo Universidad. 2012. Universidad Galileo. Web. 20 de noviembre de 2015.

Cochran-Smith, Mary. "The New Teacher Education: for Better or Worse". Educational Researcher 34 (2005): 7: 3-17. Impreso.

Chaux, Enrique. “¿Qué evalúa la Prueba de Competencias Ciudadanas?” Al Tablero 27 (2004). Impreso.

Garzón, Carlos Alberto. "Los siete retos de la educación superior”. un Periódico. (2014). Web. Abril de 2016.

Gadamer, George. Formación, entendida como cultivo de la capacidad y el talento: verdad y método. Salamanca: Ediciones Sígueme, 1984. Impreso.

Giroux, Henry. Los profesores como intelectuales: hacia una pedagogía crítica del aprendizaje. Barcelona: Paidós, 1990. Impreso.
Guzmán, Jesús Carlos. "La calidad de la enseñanza en educación superior. ¿Qué es una buena enseñanza en este nivel educativo?” Perfiles educativos 33 (2011). Web. Marzo 2016

Martínez, Enrique. Indicadores de la función didáctica. Educación y didáctica. (2011). Web. 10 de enero 2016.

Morín, Edgar, Emilio Ciurana, Raul Motta y Norma Hernández. Educar en la era planetaria. Barcelona: Gedisa, 2003. Impreso.

Moreno, Tiburcio. "La enseñanza universitaria: una tarea compleja”. Revista de la Educación Superior, ANUIES 38.3 (2009): 115-138. Web. 12 de Diciembre de 2015.

---. "Didáctica de la educación superior: nuevos desafíos en el siglo xxi”. Revista Perspectiva Educacional 50.2 (2011): 26-54. Web. Enero 5 de 2016.

Stoll, Louise, Dean Fink y Lorna Earl. It's About Learning (and it's About Time). What's in it for schools? Londres: Rutledge, 2003. Web. Abril de 2016.

Tobón, Sergio. Formación basada en competencias. Bogotá: Ecoes, 2005. Impreso. 